\title{
Modelos para la reordenación de la red de transporte público en la Comarca de Pamplona
}

\author{
José Maria Pérez Lozano \\ Tema Grupo Consultor \\ Henning Stührmann \\ Centro de estudios en transporte y movilidad (CTYM) \\ Guillermo Maldonado Riesgo \\ Tema Grupo Consultor \\ José Luis Díaz Carrero \\ Mancomunidad de la Comarca de Pamplona
}

\section{RESUMEN}

Para diseñar y evaluar alternativas la reordenación de transporte público en Pamplona se desarrollaron herramientas de modelización de dos aspectos fundamentales del sistema de transporte:

- Un modelo económico y de explotación, que relaciona la operación de las líneas con el resultado económico del operador de transporte público, y

- Un modelo de la movilidad, que relaciona la oferta en transporte público con su demanda

El primero se compone de un representación detallada de los parámetros de explotación de cada línea (kilómetros recorridos, $\mathrm{n}^{\circ}$ de expediciones, horas de conducción, material móvil), y de un modelo económico que relaciona los costes reales del operador de transporte público con parámetros de explotación. Permite inferir así el resultado económico de otras alternativas de explotación, una vez definidas con detalle su explotación.

El segundo es un modelo de la movilidad en cuatro etapas, desarrollado a partir de una encuesta domiciliaria realizada recientemente. Permite estimar la demanda de alternativas de ordenación de la red, y en particular la captada del vehículo privado.

Utilizando ambas herramientas fue posible el diseño de alternativas de ordenación de la red que, aun manteniendo prácticamente igual la subvención al sistema de transporte, incrementaban el uso del transporte público. La primera alternativa analizada corresponde a una mejora evolutiva de la actual estructura de red, mientras la segunda, basada en ejes troncales en plataforma exclusiva, constituye una mayor apuesta al transporte público. 


\section{INTRODUCCIÓN}

Para poder plantear un nuevo diseño para la red de transporte público colectivo de la Comarca de Pamplona, se ha realizado un diagnóstico del sistema de transporte público, de la movilidad y de su reciente evolución.

Este diagnostico pone de manifiesto, que la red de transporte público en la Comarca ha visto reducido su participación modal en los últimos 20 años (casi 3 puntos entre 1996 y 2013), a favor del vehículo privado. Esta evolución ha ocurrido a pesar de la calidad del servicio ofertado, en términos de cobertura, equipamiento y eficiencia, por dos hechos fundamentales:

- La dispersión urbana ocurrida en los últimos 20 años, que ha favorecido, junto con medidas de gestión de tráfico y la creación de aparcamientos, la movilidad en vehículo privado

- La falta de medidas de apoyo al transporte público, en términos de regulación semafórica, ordenación de viario y protección de itinerarios

Como conclusión pueden identificarse dos alternativas para una reordenación de la red. Una evolutiva, que optimice el sistema existente y que pueda ralentizar la perdida de participación modal, frente a otra que fortalezca los ejes troncales de la red, y que trate, junto con otras medidas de apoyo a la movilidad sostenible, de reducir la participación del vehículo privado en la movilidad de la Comarca.

Para poder diseñar y evaluar ambas alternativas se han desarrollado dos herramientas de modelización para dos aspectos fundamentales del sistema de transporte público:

- Un modelo económico y de explotación, que relaciona la operación de las líneas con el resultado económico del operador de transporte público, y

- Un modelo de la movilidad, que relaciona la oferta en transporte público con su demanda

\section{MODELO ECONÓMICO Y DE EXPLOTACIÓN}

El modelo económico y de explotación se construye para determinar los recursos necesarios (y su coste monetario) para operar una red de transporte público, sujeto al marco jurídicoeconómico de la explotación actual, formado por el contrato de concesión, el convenio laboral, y la estructura empresarial existente (material rodante disponible, sistemas IT, costes generales).

Es por ello que el modelo se ha calibrado con la cuenta de resultados de la concesión en el año 2012, para reproducirlos en base a valores unitarios de coste como:

- Coste unitario de personal de conducción.

- Precio y consumo de combustible. 
- Costes de reparaciones y mantenimientos.

- Otros consumos: lubricante, aditivos, neumáticos.

- Seguros de vehículos.

- Amortizaciones en función del número de vehículos y su precio de compra.

- Gastos financieros.

- Gastos generales de personal y de la concesión, como porcentaje de los valores anteriores.

Para ello es necesario, en primer lugar, construir un modelo de explotación que reconstruya las tres variables esenciales del modelo económico: kilómetros, horas de servicio y número de autobuses por línea en servicio. Y a partir de este desarrollar el modelo económico. Además, los modelos se completan con el modelo de demanda que se utiliza para determinar la demanda de las líneas y por tanto los ingresos del sistema, componente esencial del modelo económico.

\subsection{Modelo de explotación}

Para el modelo de explotación del transporte urbano de la Comarca de Pamplona se han graficado las líneas en servicio en 2013. Se ha graficado para tres tipos de día: laborables, sábados y festivos, tanto de invierno como de verano, obteniéndose un modelo de explotación anual. En la Figura 1 se presenta, como ejemplo, el cuadro de marchas en día laborable para la línea 16:

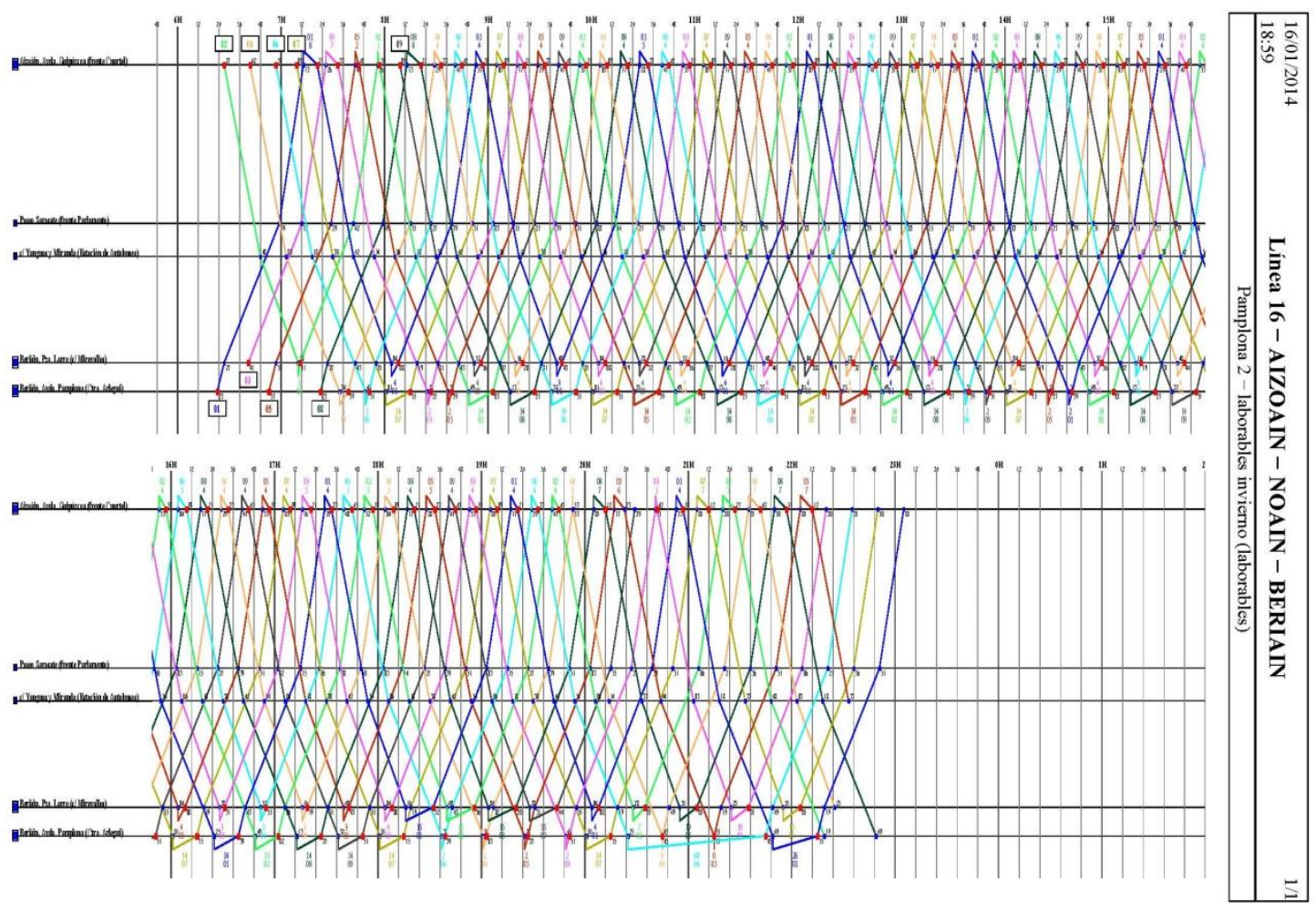

Figura 1: Cuadro de marchas Línea 16. Laborable invierno. 
La Tabla 1 recopila los resultados obtenidos a partir de este graficado, siendo de especial relevancia el número de autobuses asignado a la línea y los tiempos y distancias asociadas al servicio comercial:

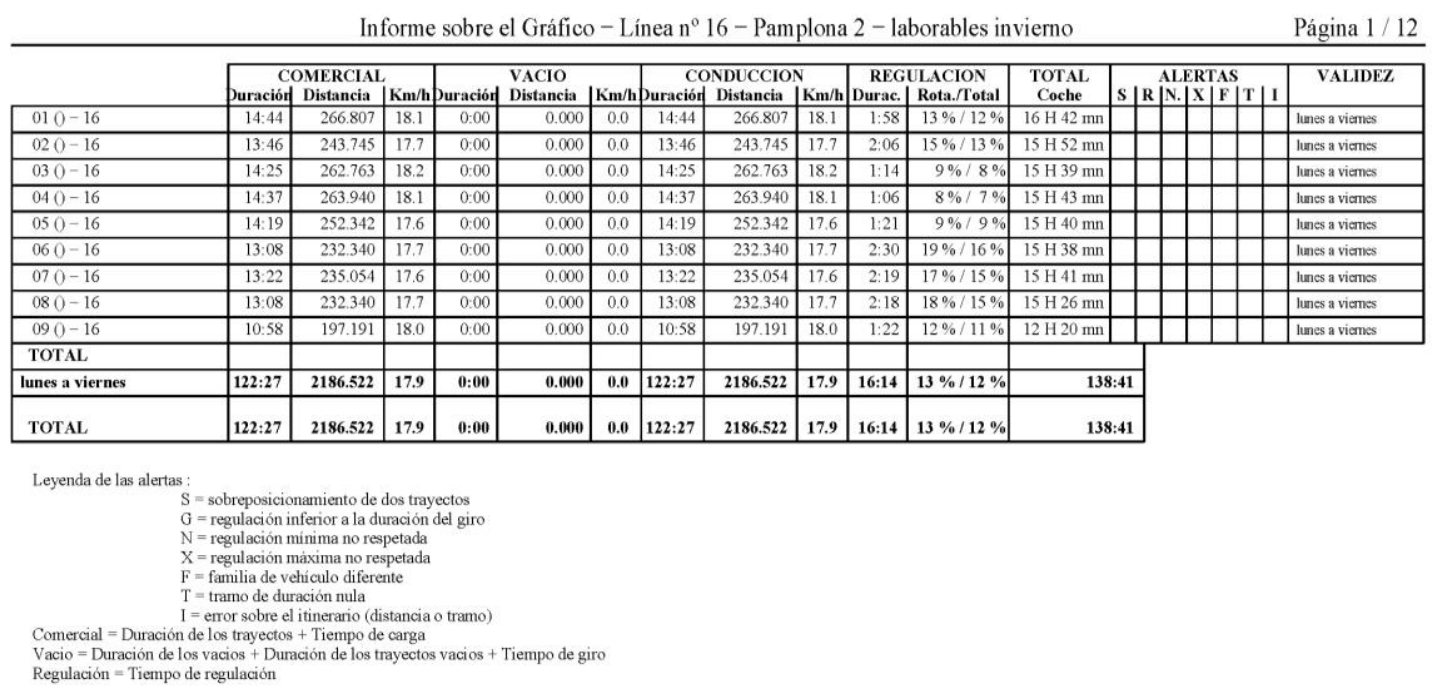

Tabla 1: Resultados Línea 16. Laborable invierno

Realizado este trabajo para todas las líneas se pueden comparar los resultados obtenidos del modelo de explotación con los reales facilitados por la concesionaria. El ajuste real respecto a 2012 demuestra unas desviaciones de un 3,2 \% para los kilómetros comerciales y de un $2,6 \%$ para las horas de servicio.

\subsection{Modelo de explotación}

Una vez elaborado y calibrado el modelo de explotación se procede a desarrollar el modelo económico que pretende reproducir la cuenta de resultados de la empresa concesionaria, en función de las magnitudes de explotación.

En concreto el modelo económico trata de replicar los valores económicos de 2012 en base a costes unitarios, aplicados a los kilómetros, horas y autobuses obtenidos del modelo de explotación, y costes indirectos producidos, fundamentalmente, por los costes del personal no conductor. Los kilómetros y horas de servicio considerados son elevados con un 5\% y un $3 \%$ respectivamente respecto de los valores arrojados por el Modelo de Explotación en concepto de toma y deje.

Según la información facilitada por la Mancomunidad algo menos del 20\% de los gastos de 
explotación corresponden a costes indirectos. A su vez, aproximadamente el $70 \%$ de estos costes indirectos corresponden a personal no conductor, como inspectores, gestión de SAE, taller, administración y dirección, mientras el restante $30 \%$ corresponde a gastos varios.

Los costes directos, que corresponden a más del $80 \%$ de los gastos de explotación, incluyen los costes de personal conductor, mantenimiento y amortización de los autobuses y su equipamiento, así como seguros, costes financieros y beneficio industrial.

Una vez calibrados los diferentes costes unitarios, el modelo económico reproduce los costes de explotación de la compañía operadora con una desviación inferior al 0,5\%.

Permite, por tanto, evaluar económicamente las redes propuestas. Para ello es necesario graficar las líneas de cada alternativa para determinar las cuatro variables básicas de coste: kilómetros, horas de servicio, expediciones y número de autobuses. El tipo de autobús, variable importante de imputación de costes directos, se define en función del número de viajeros (promedio anual) por expedición en día laborable de cada línea:

- Menos de 60 viajeros por expedición: Autobús de $12 \mathrm{~m}$.

- Entre 61 y 110 viajeros por expedición: Autobús de $18 \mathrm{~m}$.

- Más de 111 viajeros por expedición: Autobús de 24 m.

\section{MODELO DE MOVILIDAD}

Para poder evaluar el impacto de posibles modificaciones en la red de transporte público, en la demanda de viajeros que lo utiliza, se ha construido un modelo de demanda siguiendo el esquema de una modelización en cuatro etapas:

1. Generación y atracción de viajes

2. Distribución de viajes

3. Reparto modal

4. Asignación de los viajes en cada modo a las redes correspondientes

Resaltar que en la fase de reparto modal se consideran los modos

- Vehículo privado,

- Transporte público y

- Marcha a pie.

Permite, por tanto, la estimación de captaciones de demanda desde los otros modos de transporte, a raíz de mejoras en la oferta de transporte público, como podían ser incrementos de las velocidades comerciales o de las frecuencias de paso.

La Figura 2 representa el esquema general del modelo. Cabe anotar, que con excepción de la primera etapa, estas se condicionan mutuamente. Así, por ejemplo, la distribución de 
viajes influye en el reparto modal, que a su vez impacta los modelos de asignación.

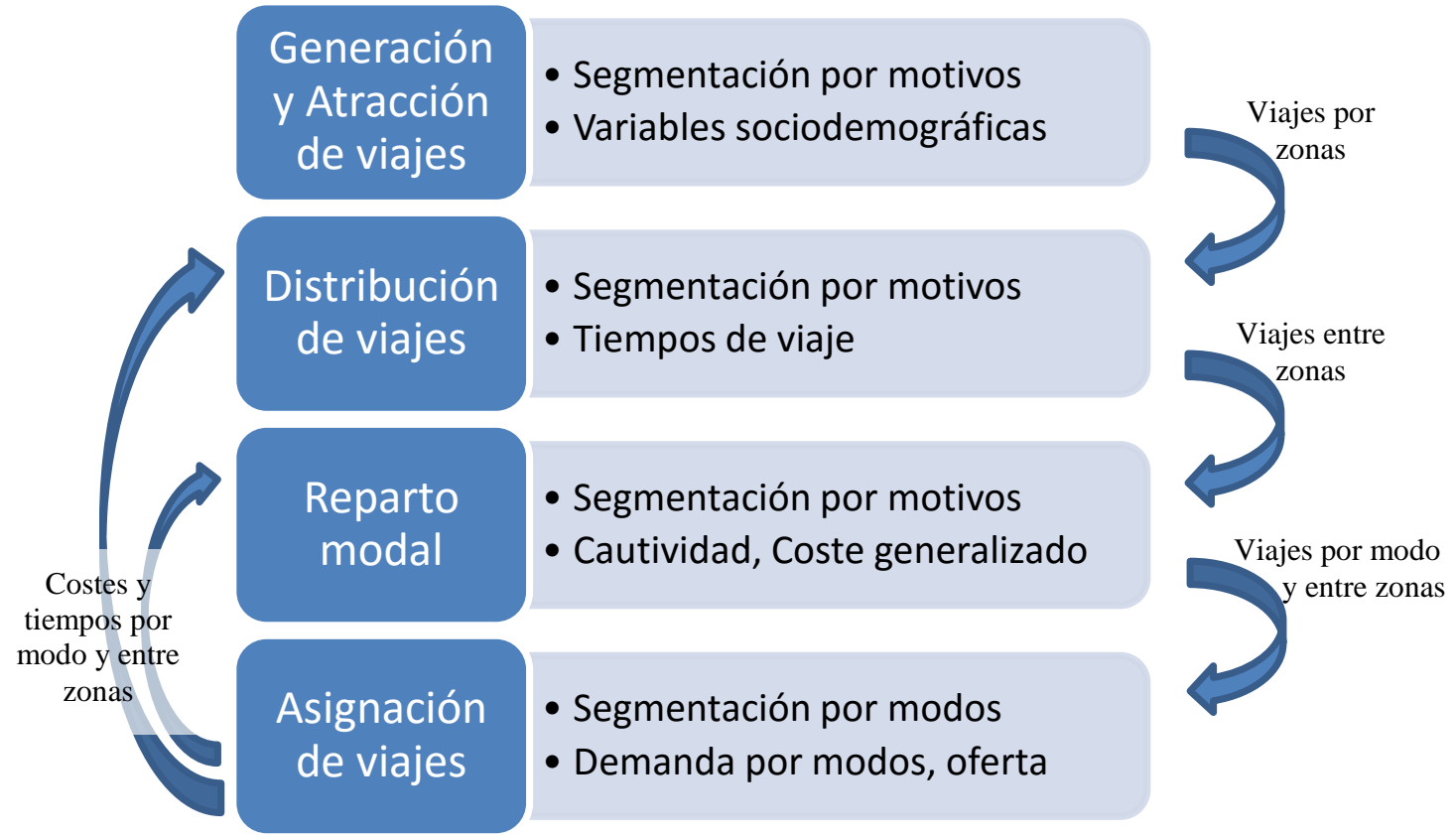

Figura 2: Esquema general del modelo de cuatro etapas

De estos últimos se obtienen los tiempos de viaje, que condicionan tanto los modelos de distribución como de reparto modal. Es por ello que el proceso de modelización se inicia con una primera estimación de los tiempos y costes entre zonas, derivada de la asignación "directa" de los viajes obtenidos en la encuesta.

\subsection{Modelos de generación y atracción de viajes}

Para el desarrollo de los modelos de generación y atracción se ha partido de los viajes recopilados en la Encuesta Domiciliaria de Movilidad. La modelización se ha segmentado según los siguientes motivos de viaje:

- Movilidad obligada:

- Trabajo

○ Estudios

- Movilidad no-obligada: Otros motivos

Los modelos reflejan, al igual que la encuesta, la movilidad de un día medio laborable.

\subsubsection{Generación de viajes}

Para el desarrollo de modelos de generación de viaje, se ha optado por el uso de modelos de clasificación cruzada, aplicadas de forma desagregada a la información recopilada en la encuesta domiciliaria. Para ello se ha calculado el número diario de viajes según motivos de viaje para cada encuestado, y se ha procedido a analizar el promedio de desplazamientos para grupos o clases de usuarios según determinadas características de la persona o del hogar 
en que reside.

La Figura 3 muestra la segmentación de los viajes realizados por motivo trabajo, según las características socioeconómicas. Se observa que más del 90\% de los viajes es realizado por personas ocupadas, con al menos un coche en el hogar. Es, por tanto, este segmento de la demanda que merece mayor desagregación.

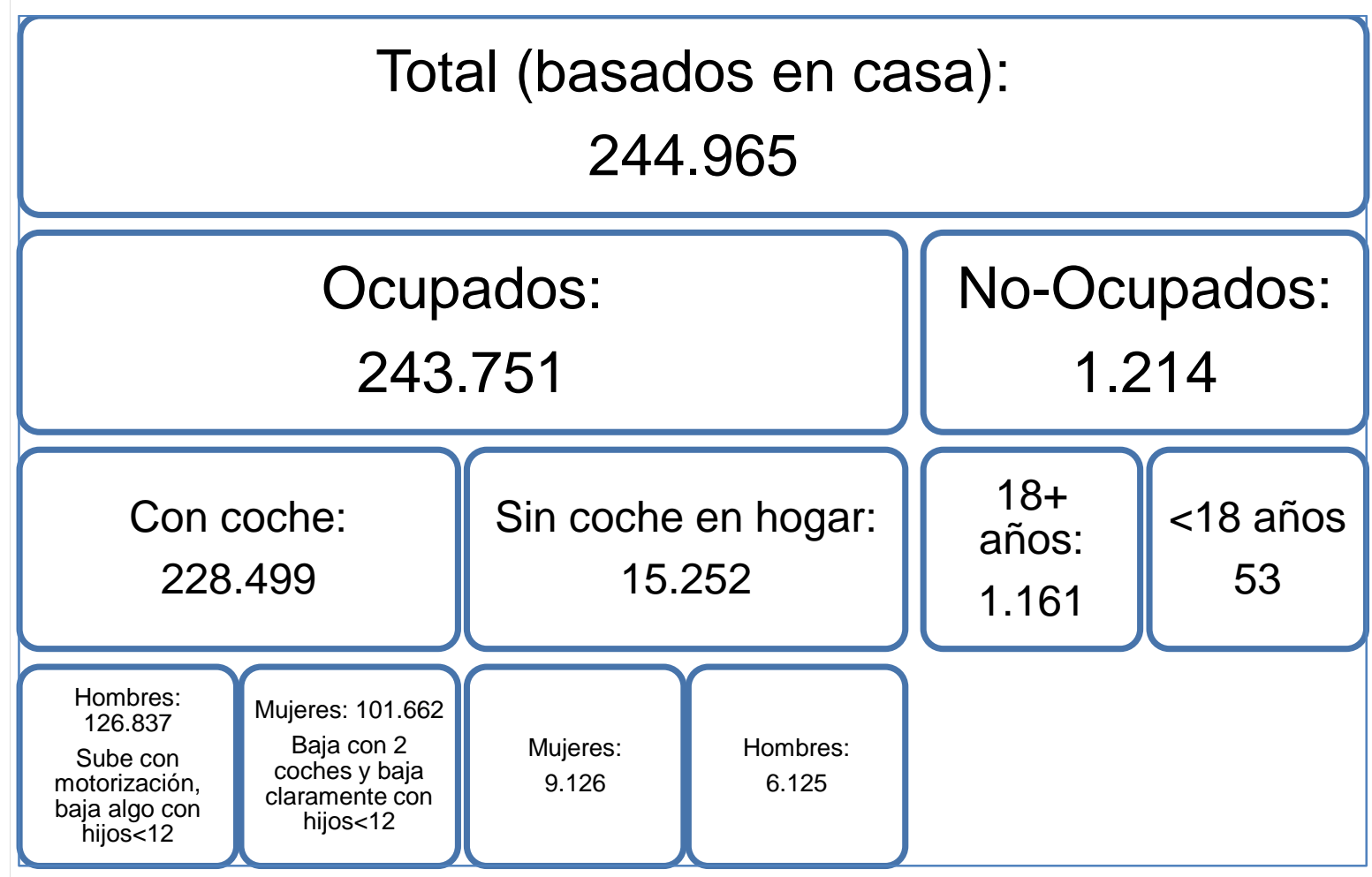

Figura 3: Segmentación de los viajes por motivo trabajo

Un 55\% de los viajes es realizado por hombres y el $45 \%$ por mujeres. Analizando las tasas de movilidad dentro de estos últimos dos segmentos se observan variaciones (ver Tabla 2) en función de la motorización y del número de hijos menores de 12 años. Mientras los segundos reducen la movilidad tanto de hombres como de mujeres (donde el efecto es más pronunciado), la motorización tiene efectos opuestos en ambos sexos: el segundo coche aumenta la movilidad de hombres, pero la reduce en el caso de las mujeres.

Dado la muestra reducida de viajes de hogares sin coches, este grupo solamente se ha segmentado entre hombres y mujeres, mientras del grupo de no-ocupados se han separado los escasos casos de menores de 18 años, cuya tasa de viaje se ha asumido en cero.

Como se puede observar en la Tabla 2, la clasificación cruzada ha dado lugar a 16 segmentos socioeconómicos, con tasas de viaje que varían, frente a una media de 0,77 viajes persona, desde cero hasta 1,92 viajes persona. Entre los ocupados, que realizan la práctica totalidad de los viajes por motivo trabajo, las tasas varían entre 1,28 viajes persona (Mujeres, con $2 \mathrm{o}$ más hijos y 2 o más coches en el hogar) a 1,92 viajes persona (Hombres, con 2 o más hijos, 
y sin vehículo en el hogar).

\begin{tabular}{|c|c|c|c|c|c|}
\hline & Segmento & Sexo & Coches & Hijos & Viajes/persona \\
\hline \multirow{14}{*}{ 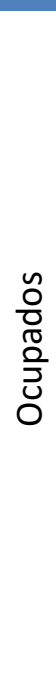 } & \multirow{12}{*}{$\begin{array}{c}\text { Con Coche } \\
\text { (228.499 viajes) }\end{array}$} & Hombre & 1 & 0 & 1,8394 \\
\hline & & Hombre & 1 & 1 & 1,8874 \\
\hline & & Hombre & 1 & 2 o más & 1,6285 \\
\hline & & Hombre & 2 o más & 0 & 1,9178 \\
\hline & & Hombre & 2 o más & 1 & 1,7978 \\
\hline & & Hombre & 2 o más & 2 o más & 1,8541 \\
\hline & & Mujer & 1 & 0 & 1,8566 \\
\hline & & Mujer & 1 & 1 & 1,6098 \\
\hline & & Mujer & 1 & 2 o más & 1,1839 \\
\hline & & Mujer & 2 o más & 0 & 1,8643 \\
\hline & & Mujer & 2 o más & 1 & 1,4687 \\
\hline & & Mujer & 2 o más & 2 o más & 1,2811 \\
\hline & \multirow{2}{*}{$\begin{array}{c}\text { Sin Coche } \\
\text { (15.2525 viajes) }\end{array}$} & Hombre & 0 & & 1,8471 \\
\hline & & Mujer & 0 & & 1,7042 \\
\hline \multicolumn{5}{|c|}{ No-Ocupados > 18 años (1.161 viajes) } & 0,0089 \\
\hline \multicolumn{5}{|c|}{ No-Ocupados $<18$ años (53 viajes) } & 0 \\
\hline
\end{tabular}

\section{Tabla 2: Tasas de generación de viajes por motivo trabajo según segmento}

Para obtener y valorar los resultados de los modelos para cada uno de los tres motivos (trabajo, estudio y movilidad no-obligada), se ha procedido, para cada zona de transporte a:

- Determinar, con ayuda de la encuesta y del padrón, el número de residentes en cada clase,

- Multiplicar este dato con la tasa calculada en el presente análisis, para obtener el total de viajes por motivo trabajo generado:

$$
V_{i}=\sum_{c} P_{i, c} \cdot T_{i, c}
$$

donde i: zona, c: clase, P: población, T: tasa de generación de viaje

- Compararlo con el dato de viajes por motivo trabajo observado en la encuesta de movilidad.

Los tres modelos de generación reproducen de forma adecuada los viajes generados por zona de transporte observados en la encuesta. En todo caso, y para evitar llevar los desajustes del año base a las proyecciones de demanda en los años horizonte, la aplicación de los modelos en los años horizontes se realiza de forma incremental, es decir los viajes del año horizonte $\mathrm{V}^{*}$ se obtienen como suma entre los viajes observados en el año base, y los viajes generados por la variación de las poblaciones en cada clase c:

$$
\mathrm{V}_{\mathrm{i}}^{*}=\mathrm{V}_{\mathrm{i}}+\sum_{\mathrm{c}} \Delta \mathrm{P}_{\mathrm{i}, \mathrm{c}} \cdot \mathrm{T}_{\mathrm{i}, \mathrm{c}}
$$

donde $\Delta P_{i, c}=P_{i, c}^{*}-P_{i, c}$ es la variación de la población de la zona i y clase c en el año horizonte con respecto al año base. 


\subsubsection{Atracción de viajes}

Para la modelización de la atracción de viajes se han utilizado modelos agregados de la demanda atraída. Se trata, por tanto, de explicar los viajes atraídos en cada zona en función de variables socioeconómicas (p.e. habitantes o puestos de trabajo) o de usos urbanos (p.e. colegios, hospitales o centros comerciales). Para ello se han calibrado modelos de regresión lineal múltiple.

A igual que en el caso de la generación, los modelos se han calibrado diferenciando los motivos trabajo, estudios, y la movilidad no-obligada.

Para el primer motivo las variables explicativas son los números de puestos de trabajo en el sector de servicios por un lado, y del resto de los sectores por el otro, con coeficientes de 1,8 viajes por día y puesto y de 1,7 viajes por día y puesto, respectivamente.

Las variables explicativas para viajes por motivo estudios han sido los números de plazas de educación superior, de educación escolar, y de extra-escolar (por ejemplo academias), con coeficientes de 2,1 viajes por día y plaza, 2 viajes por día y plaza, y de 1,8 viajes por día y plaza.

Finalmente, para viajes con motivo no-obligado se han empleado como variables explicativas la población, el número de puestos de trabajo en el sector de servicios y dos variables dummy para reproducir la concentración de actividad comercial y administrativa en las zonas centro de los principales municipios de la comarca, así como la actividad de zonas comerciales y de ocio fuera de estas. Los coeficientes son 0,6 viajes por día y habitante, y de 3 viajes por día y puesto, respectivamente.

Los tres modelos reproducen de forma adecuada los viajes atraídos por zona de transporte observados en la encuesta. A igual que en el caso de la generación, la aplicación de los modelos en los años horizontes se realiza de forma incremental

\subsection{Modelos de distribución espacial de viajes}

Estos modelos tratan de reproducir la distribución de los viajes generados en una zona entre las zonas de transporte en que está dividido el ámbito del estudio. En primer lugar cabe recordar que, a diferencia del caso de generación y atracción, no se dispone de observaciones (y menos estadísticamente significantes) en la gran mayoría de las relaciones. Sin embargo, si asociamos los tiempos de desplazamiento a cada par de origen destino, sí se puede determinar:

- El tiempo medio de desplazamiento

- La distribución de los viajes en función del tiempo de recorrido

- La demanda en la mayoría de las relaciones entre macrozonas 
La Figura 4 recopila en forma de histogramas la distribución de los viajes totales y segmentados por motivos (trabajo, estudios y no-obligados) en función del tiempo medio de viaje. Para el total de la demanda se observa un tiempo medio de 17,7 min, una desviación estándar de 14,5 min y una distribución con una cola larga a la derecha. Destacar que el $15,8 \%$ de los viajes corresponden a viajes intrazonales a los que se ha asignado un tiempo de menos de un minuto ya que no se dispone de estimación de tiempo medio para ellos.

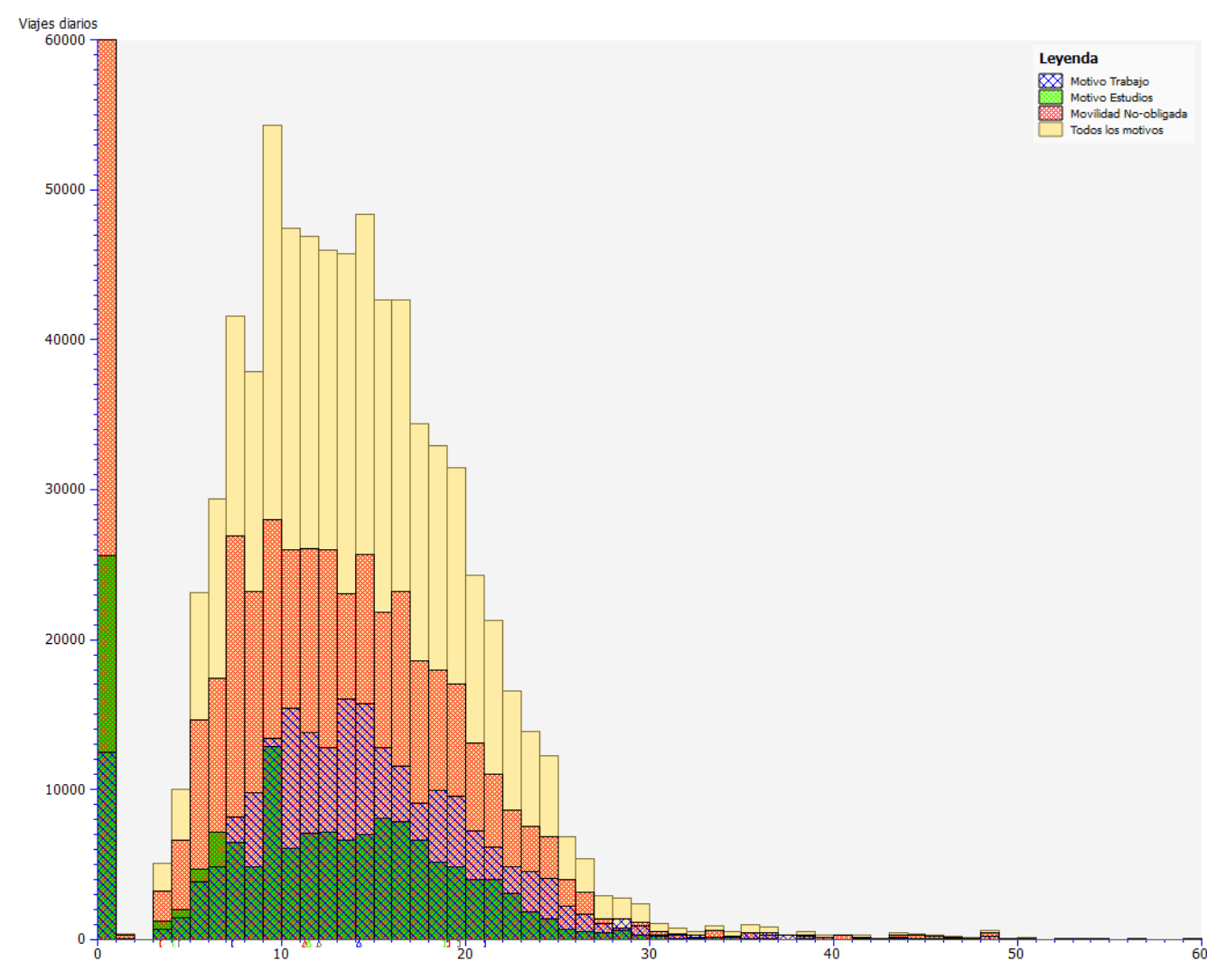

Figura 4: Distribución de la demanda observada según tiempo medio de viaje

Cabe resaltar las diferencias en la distribución según motivos de viaje, tanto en cuanto a tiempos medios como en la forma de la distribución, como puede deducirse de la Tabla 3 que resume los parámetros más importantes:

\begin{tabular}{|c|c|c|c|c|}
\hline Motivo & $\begin{array}{l}\text { Tiempo medio } \\
\text { [min] }\end{array}$ & $\begin{array}{c}\text { Desviación } \\
\text { estándar [min] }\end{array}$ & $\begin{array}{c}\text { Tiempo } \\
\text { máximo }[\mathrm{min}]\end{array}$ & $\begin{array}{c}\text { \% Viajes } \\
\text { intrazonales }\end{array}$ \\
\hline Todos & 12,2 & 7,6 & 70,3 & $15,80 \%$ \\
\hline Trabajo & 14,2 & 6,9 & 70,3 & $5,70 \%$ \\
\hline Estudio & 11,5 & 7,4 & 54,0 & $17,20 \%$ \\
\hline Movilidad no-obligada & 11,3 & 7,9 & 57,1 & $19,80 \%$ \\
\hline
\end{tabular}

Tabla 3: Características de las distribuciones de viajes según motivo. 
De ello se deduce la necesidad de ajustar modelos de distribución diferenciados por motivo.

Utilizando por un lado el tiempo medio de desplazamiento y por el otro los flujos entre macrozonas pueden calibrarse modelos de distribución de tipo gravitatorio con la siguiente formulación:

\section{Donde}

$$
V_{i j}=a_{i} \cdot b_{j} \cdot c_{m k} \cdot \frac{o_{i} \cdot D_{j}}{f\left(T_{i j}\right)}
$$

$\mathrm{V}_{\mathrm{ij}}$ es la matriz de viajes entre las zonas i y j, denominada generalmente la matriz "sintética" $a_{i}, b_{j}, c_{m k}$ son coeficientes de balanceo en la zona de origen $i$, la zona de destino $j$ y de la relación entre macrozonas $\mathrm{mk}$, con $\mathrm{i} \in \mathrm{m} ; \mathrm{j} \in \mathrm{k}$.

$\mathrm{O}_{\mathrm{i}}, \mathrm{D}_{\mathrm{j}}$ son los viajes con origen en $\mathrm{i}$, respectivamente destino en $\mathrm{j}$

$f\left(T_{i j}\right)$ es una función del tiempo de viaje $T_{i j}$ entre i y j. Aquí se ha elegido una función de coste exponencial, $f\left(T_{i j}\right)=e^{\theta \cdot T_{i j}}$

En el caso del motivo trabajo, como resultado de la calibración se obtiene un coeficiente $\theta$ de 0,0560. El tiempo promedio de la matriz sintética es, con 11,81 minutos, casi idéntico al observado, de 11,82 minutos.

En el caso del motivo estudios, como resultado de la calibración se obtiene un coeficiente $\theta$ de 0,0766. El tiempo promedio de la matriz sintética es, con 14,23 minutos, casi idéntico al observado, de 14,22 minutos.

En el caso los viajes no-obligados, como resultado de la calibración se obtiene un coeficiente $\theta$ de 0,0927 . El tiempo promedio de la matriz sintética es, con 12,2 minutos, idéntico al observado.

A modo de ejemplo la Figura 5 recopila la distribución según tiempo de viaje de la matriz modelizada por motivo trabajo en comparación con la distribución de la matriz observada. Puede constatarse una coincidencia elevada. 


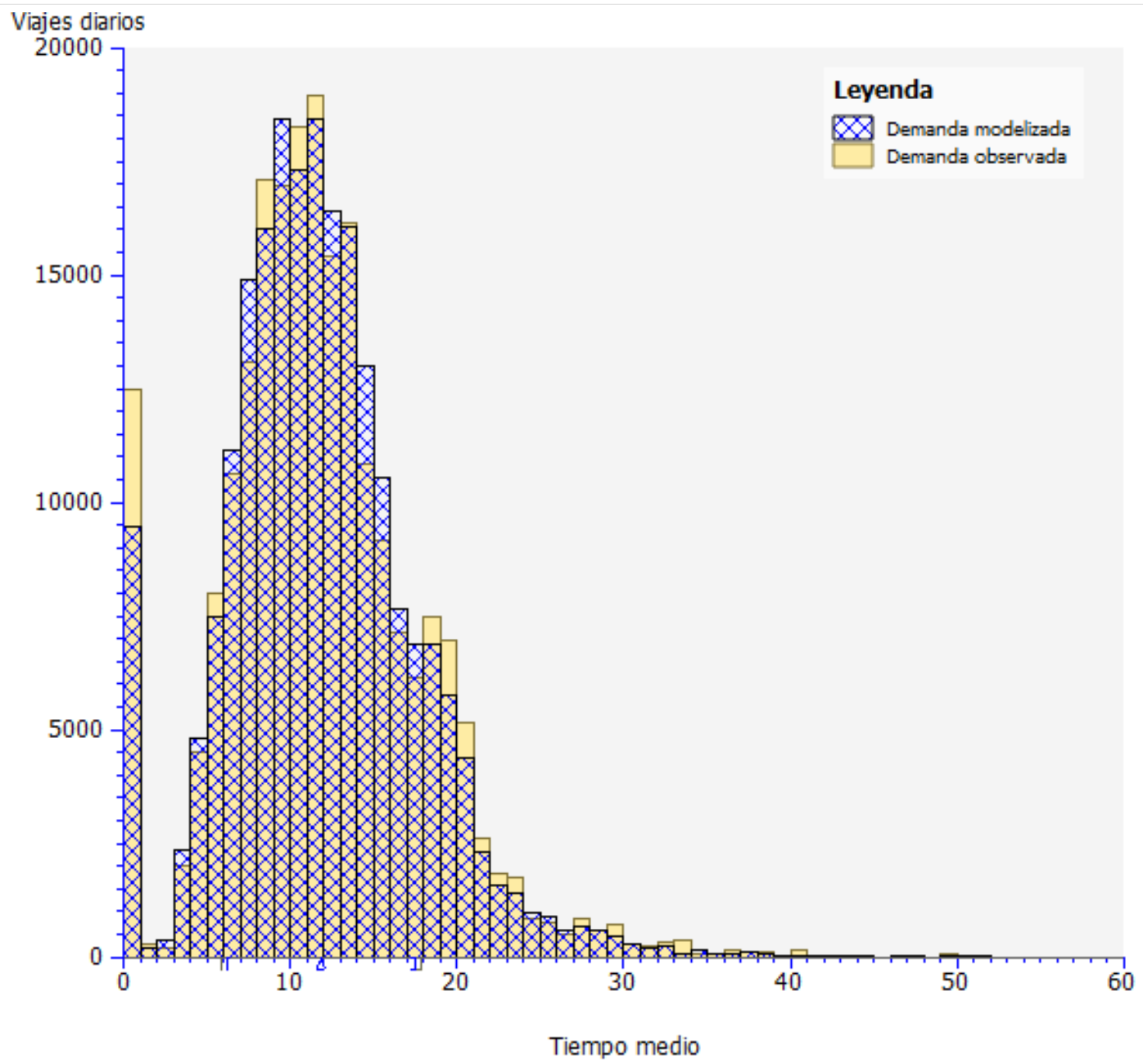

Figura 5: Distribución de los viajes por motivo trabajo según tiempo. Matriz modelizada vs. observada.

\subsection{Modelos de reparto modal}

Para el desarrollo de los modelos de reparto modal se ha optado por utilizar modelos logit, en principio de tipo jerárquico, procediendo a su calibración con la información desagregada de las encuestas domiciliarias. No obstante y para facilitar su aplicación a escenarios horizonte, en la elección de las variables explicativas se ha tenido en cuenta la factibilidad de proyectarlas.

Los modos de transporte recopilados en la encuesta domiciliaria se han agrupado en cuatro modos:

- Vehículo privado
○ Como conductor
○ Como acompañante

- Autobús

- Marcha a pie 
Como en el caso de los modelos de generación/atracción y distribución, también aquí la demanda se ha segmentado según los siguientes motivos:

- Movilidad obligada:

$$
\begin{array}{ll}
\circ & \text { Trabajo } \\
\circ & \text { Estudios }
\end{array}
$$

- Movilidad no-obligada: Otros motivos

En el proceso de calibración se han obtenido, para los motivos estudios y no-obligados, modelos jerárquicos que agrupan los dos tipos de usuarios de vehículo privado (conductor y acompañante) en un solo nido, mientras para el motivo trabajo el modelo elegido es multinomial.

Las funciones de utilidad incluyen, en todos los modos, el tiempo total de viaje, el coste y una constante modal diferente según sexo del encuestado. Los coeficientes de tiempo y coste son los mismos en todos los modos. Con ello el valor de tiempo varía según motivo, pero no según modo de transporte.

El modo a pie no tiene coste ni constante modal al considerarse el modo más universal. Adicionalmente, en las funciones de utilidad del transporte público se utilizan el tiempo de acceso, el número de transbordos y la frecuencia de paso. En el caso del vehículo privado se utiliza una penalización adicional para viajes con destino en zonas con aparcamiento regulado. Finalmente, cabe resaltar que el modo coche conductor no está disponible para menores de 18 años o personas sin carnet.

Los tres modelos reproducen de forma adecuada los patrones de reparto modal observados en la encuesta. Los valores de tiempo implícitos son algo mayores que $9 €$ por hora para el motivo trabajo, de algo menos de $3 €$ por hora para el motivo estudios, y de poco más de $2,50 €$ por hora para los motivos no-obligados.

La aplicación de los modelos de reparto calibrados de forma desagregada con las encuestas de movilidad se realiza de forma incremental. Con ello los cambios en el reparto modal entre la situación base y un escenario con oferta (y/o características socioeconómicas modificadas) son resultado de los cambios de utilidad y del reparto modal en el año base:

$$
\mathrm{p}_{\mathrm{m}}=\frac{\mathrm{p}_{\mathrm{m}}^{0} \exp \left(\theta \Delta \mathrm{U}_{\mathrm{m}}\right)}{\sum_{\mathrm{q}} \mathrm{p}_{\mathrm{q}}^{0} \exp \left(\theta \Delta \mathrm{U}_{\mathrm{q}}\right)} ; \mathrm{m} \in \mathrm{M}, \mathrm{q} \in \mathrm{M}
$$

Donde

$\mathrm{p}_{\mathrm{m}}, \mathrm{p}_{\mathrm{m}}^{0}$ son las cuotas modales del modo $\mathrm{m}$ en el escenario a investigar y en la situación base $\Delta \mathrm{U}_{\mathrm{m}}=\mathrm{U}_{\mathrm{m}}-\mathrm{U}_{\mathrm{m}}^{0}$ es la variación de utilidad entre el escenario y la situación base $M$ es el conjunto de modos de transporte 


\subsection{Modelos de red y asignación}

Se ha partido de una red multimodal, facilitada por la Mancomunidad de la Comarca de Pamplona, que ha sido revisada y detallada donde fuese preciso. Cabe resaltar la interacción entre la red codificada para los desplazamientos a pie, y la red de transporte público, donde estos desplazamientos constituyen el acceso a la red de autobús y la dispersión desde esta a los destinos finales. Las modificaciones más relevantes han sido las siguientes:

- Introducción de la nueva zonificación: centroides y conectores entre estos y el grafo de la red

- Re-codificación de las líneas según los últimos itinerarios facilitados por la Mancomunidad. Se han introducido con codificación explícita de paradas y sus velocidades se han ajustado a lo observado en los trabajos de campo

- Creación de itinerarios peatonales: ascensores, zonas peatonales, principales parques y, sobre todo, en ambos sentidos de circulación en calles de sentido único.

La red resultante consiste de 152 centroides, conectados mediante 630 arcos (un solo sentido) al grafo de la red, formado por 1.593 nodos, 4.204 arcos (un solo sentido) y 62 itinerarios de transporte público (correspondientes a 23 líneas). Aproximadamente el 17\% de los arcos (723) corresponden a viario exclusivo del peatón.

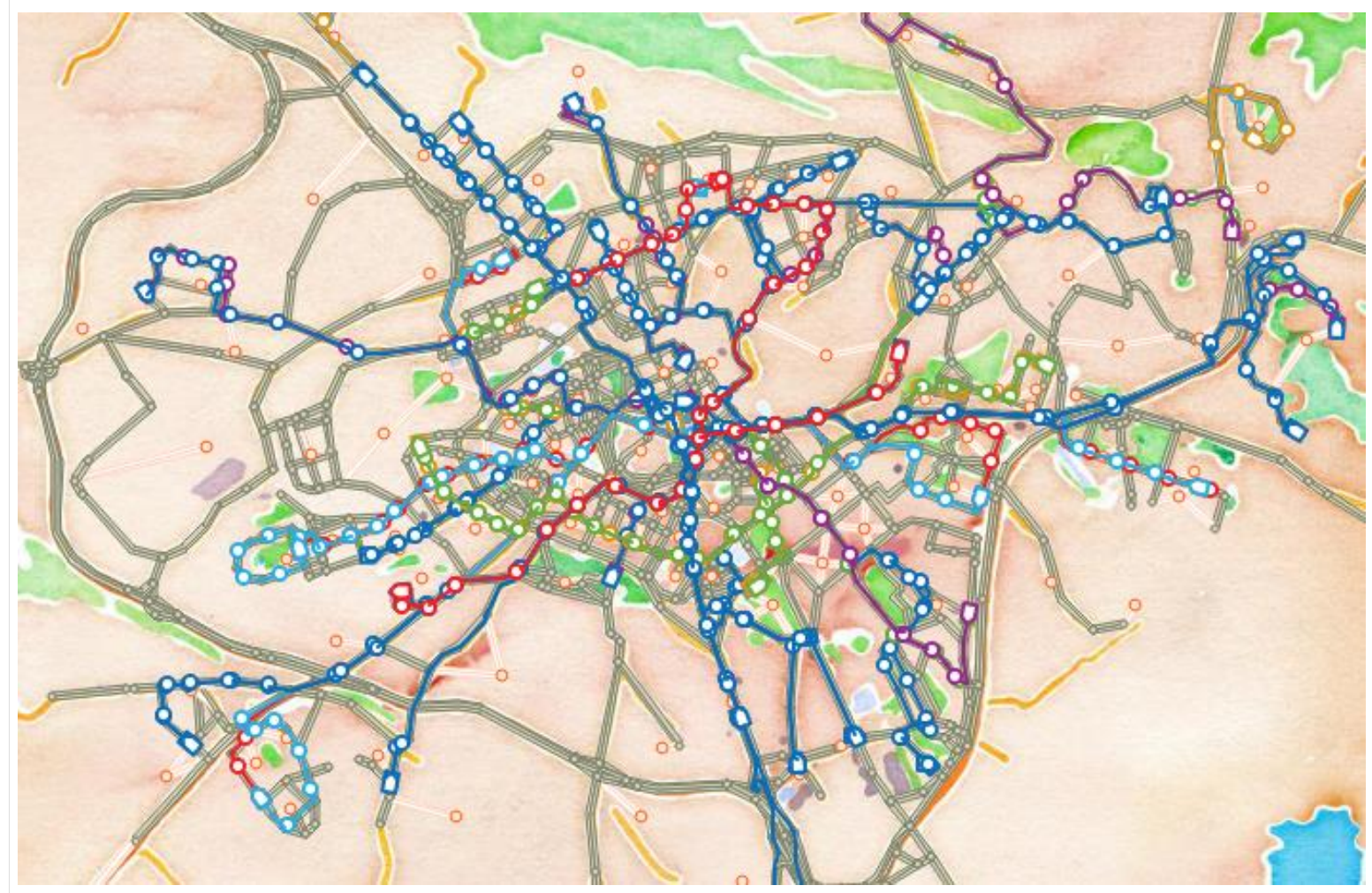

Figura 6: Grafo de la red modelizada

Las asignaciones en transporte público se han realizado con el módulo de asignación extendida de transporte público de Emme. Los algoritmos utilizados se basan en el principio 
de la estrategia óptima, pero permiten ajustes adicionales, como por ejemplo, en la distribución entre conectores en origen.

De la asignación se extraen, a parte de los volúmenes de viajes en cada segmento de línea, y en los arcos de la red de acceso/dispersión, los tiempos de viajes, diferenciando entre

- El tiempo de espera

○ Primera espera

○ Esperas en etapas siguientes

- Tiempo abordo

- Tiempo de acceso y dispersión (incluido eventuales etapas intermedias a pie)

- Número de transbordos

Cabe resaltar que estos datos corresponden, para cada relación entre dos zonas de transporte, al promedio de las diferentes rutas que componen la estrategia óptima. Son estas matrices de tiempo que alimentan los modelos de reparto modal.

La asignación de la matriz de transporte público del año base (obtenida de la encuesta domiciliaria, y ajustada a los viajes detectados en las encuestas a bordo de las líneas de autobús), reproduce de manera satisfactoria las cancelaciones de una semana media de septiembre.

\section{RESULTADOS}

El trabajo realizado se alinea con los objetivos iniciales planteados por la Mancomunidad y han permitido diseñar dos alternativas de red para motivar un cambio modal en el sistema de transporte público. El trabajo parte de unos datos de campo realizados con suficiente calidad como para garantizar que los resultados de modelización cumplen con los parámetros marcados.

Ambas redes se pueden considerar como elementos diferenciados o como etapas de un plan director de red, en el que se puedan ir incorporando cambios de forma secuencial en la dirección de conseguir una red troncal futura.

Para la Mancomunidad, el sistema de modelización descrito, deja implantado un modelo de movilidad que permite gestionar los cambios futuros de red de una forma coordinada y permite plantear cambios tangibles hacia los órganos decisores en la idea de plantear futuras estrategias de cambio modal. 